\title{
Nonlinear Robust Control of Tendon-Driven Robot Manipulators
}

\author{
Beytullah Okur • Orhan Aksoy • \\ Erkan Zergeroglu $\cdot$ Enver Tatlicioglu
}

Received: 22 November 2013 / Accepted: 25 September 2014 / Published online: 11 October 2014

(C) Springer Science+Business Media Dordrecht 2014

\begin{abstract}
This work addresses the position tracking control problem for tendon-driven robotic mechanisms in the presence of parametric uncertainty and additive external disturbances. Specifically, a full state feedback nonlinear robust controller is proposed to tackle the link position tracking problem for tendondriven robot manipulators with uncertain dynamical system parameters. A robust backstepping approach has been utilized to achieve uniformly ultimately bounded tracking performance despite the lack of exact knowledge of the dynamical parameters and presence of additive but bounded disturbance terms. Stability of the overall system is proven via Lyapunov based arguments. Simulation studies performed
\end{abstract}

This research is supported by Grants of the Scientific and Technological Research Council of Turkey, TUBITAK Project No: 112E561

B. Okur · O. Aksoy · E. Zergeroglu $(\triangle)$

Department of Computer Engineering, Gebze Institute

of Technology, Gebze, Kocaeli 41400, Turkey

e-mail: ezerger@bilmuh.gyte.edu.tr

E. Tatlicioglu

Department of Electrical \& Electronics Engineering,

Izmir Institute of Technology, Izmir 35430, Turkey

O. Aksoy

DSYSTM Havelsan A.S., Istanbul 34890, Turkey

B. Okur

Department of Mechatronics Engineering, Yildiz

Technical University, Istanbul 34349, Turkey on a two link planar robot manipulator driven by a six tendon mechanism are presented to illustrate the effectiveness and viability of the proposed approach.

Keywords Nonlinear systems · Robot manipulators · Tendon driven system systems $\cdot$ Robust control

\section{Introduction}

The idea of separating the actuators from the links of the robot and remote actuating each joint has always attracted the robot designers as this will reduce the bulk and extra inertia from the mechanical system. Among other remote power transmitting methods, tendon-driven transmission systems present a less noisy, clean (as they do not require lubrication), and shock absorbent characteristics. Therefore tendondriven mechanisms have been used in the design of both small [1-3] and large robot manipulators [4-6]. However the use of tendon-driven actuation has been more popular in dexterous hands [2, 3, 7] as the resultant task-space motion in robotic hand designs does not need to be accurate and relatively simple controllers can handle desired objectives. For robot manipulators where the main aim is to track a task-space trajectory as closely as possible, the use of tendon-driven mechanisms are limited. We believe that the main reason for this is the additional complexity of the overall system dynamics. For better control performance, it is necessary to consider the 
system dynamics in the controller design. However, when the dynamics of the power transmission system is added to the system model, the control design problem becomes more complicated mostly due to the addition of extra dynamics and therefore additional uncertainties. On top of this, for an acceptable tracking performance, tendon elasticity in the transmission system, specific to tendon-driven systems, has to be considered in the controller design $[5,8]$.

Aside from the classical linear control approaches (see [9, 10], and the references therein), to our best knowledge, controller formulations including the system dynamics for tendon-driven robot manipulators are penurious in the literature. To name some; the development and performance of an inner-loop adaptive and robust outer-loop controller was investigated in [11], while in [12], robotic mechanisms with redundant tendons were investigated and in [13], authors propose a puller-follower controller both compliant and non-compliant antagonistic tendon drives in robotic systems. In [5], Kobayashi and Ozawa presented an adaptive and an adaptive neural network based controller for tendon-driven robotic mechanisms with elastic tendons. In [14], Nakayama and Fujimoto tackled the tracking control of tendondriven robots by applying the delayed reflexive force feedback. In [15, 16], Haiya et al. proposed a controller for multiple degree-of-freedom tendon mechanisms using nonlinear springs with hysteresis characteristics like stiffness adjustable tendons. For the proposed controllers, error of the equation of spring was estimated by a disturbance observer and compensated by utilizing the estimated disturbance. In [17], Wimbock et al. proposed an application of the Immersion and Invariance type framework to tendondriven systems with variable stiffness. Among the above cited work, the only work that considered the uncertainties in the system dynamics was given in [5], however the proposed adaptive controller required the measurement of the second and third time derivatives of link position measurements (see assumption 2 of [5]) which are usually not available.

Review of the past research on control of tendondriven robot manipulators has revealed that almost all of the control designs required accurate knowledge of the system model along with exact knowledge of model parameters, and according to the authors' best knowledge, the only work considered parametric uncertainties was [5] which utilized link acceleration, and jerk measurements. In this study, we aim to design a robust controller that does not require acceleration and jerk measurements. Specifically, for the restrictive case of full state feedback (i.e., position and velocity information of both the actuators and robot links and tension measurements of each tendon) being available, and the robot dynamic model and the actuator dynamics including uncertain parameters is considered. Adding the dynamics of the power transmission system and considering tendon elasticity yield a complicated dynamic model, and the resulting system dynamics mandates the use the backstepping technique twice (unlike our previous work [18], where robust backstepping was applied only once). After fusing the backstepping design procedure with Lyapunov-type analysis tools, we design the auxiliary backstepping control inputs and the control input applied to the actuators. The stability analysis ensures the boundedness of all the signals under the closedloop operation and uniform ultimate boundedness of the link position tracking error. Our theoretical results are verified via numerical simulations.

The rest of the work is organized in the following manner. The dynamical model of tendondriven manipulators along with model properties are presented in Section 2. Controller formulation and the corresponding stability analysis are given in Sections 3 and 4, respectively. Section 5 contains the numerical simulations performed on a two link manipulator driven by a six tendon mechanism, and concluding remarks are given in Section 6.

\section{System Dynamics and Model Properties}

The dynamics of an $n$ degree-of-freedom robot manipulator driven by an $m$-tendon mechanism have the following form [5]

$$
\begin{aligned}
M(q) \ddot{q}+V_{m}(q, \dot{q}) \dot{q} & +F_{d} \dot{q}+G(q)+d_{1} \\
& =-J_{j}^{T}(q) f_{t}(l) \\
J \ddot{\theta}+B \dot{\theta} & +R_{a} f_{t}(l)+d_{2}=\tau_{a} \\
i & =J_{j}(q) \dot{q}+R_{a} \dot{\theta}
\end{aligned}
$$

where $q(t), \dot{q}(t), \ddot{q}(t) \in \mathbb{R}^{n}$ represent the link position, velocity and acceleration vectors, respectively, $\theta(t), \dot{\theta}(t), \ddot{\theta}(t) \in \mathbb{R}^{m}$ represent the actuator position, velocity and acceleration vectors, respectively, 
$l(t), i(t) \in \mathbb{R}^{m}$ are the $m$ - dimensional tendon expansion vector and its time derivative, $M(q) \in \mathbb{R}^{n \times n}$ denotes the link inertia effects, $V_{m}(q, \dot{q}) \in \mathbb{R}^{n \times n}$ represents the centripetal Coriolis effects, $G(q) \in \mathbb{R}^{n}$ denotes the gravitational terms related to the robot, $F_{d} \in \mathbb{R}^{n \times n}$ is the constant diagonal link viscous friction matrix, $d_{1} \in \mathbb{R}^{n}$ and $d_{2} \in \mathbb{R}^{m}$ are used to represent the bounded (with known upper bounds) disturbance vectors, $J, B \in \mathbb{R}^{m \times m}$ are the diagonal actuator inertia and actuator viscous friction matrices, respectively, $R_{a} \in \mathbb{R}^{m \times m}$ is the diagonal matrix containing the known radius of pulleys mounted on each actuator, $J_{j}(q) \in \mathbb{R}^{m \times n}$ is the known Jacobian matrix that maps the joint space to the tendon expansion space, $f_{t}(l) \in \mathbb{R}^{m}$ is the known vector of tendon tensile forces generated by the tendon expansions, and finally $\tau_{a}(t) \in \mathbb{R}^{m}$ is the control input vector applied the actuators. A schematic representation of the system dynamics is presented in Fig. 1. The dynamic equations of Eq. 1 exhibit the following useful properties, which will be utilized in the controller development and the subsequent stability analysis.

Property 1 The inertia matrix can be bounded from above and below by the following inequalities [21]

$m_{1} I_{n} \leq M(q) \leq m_{2} I_{n}$

where $m_{1}$ and $m_{2}$ are positive constants, and $I_{n}$ is the $n \times n$ identity matrix. Likewise the inverse of the inertia matrix can be bounded as follows

$\frac{1}{m_{2}} I_{n} \leq M^{-1}(q) \leq \frac{1}{m_{1}} I_{n}$.
Property 2 The inertia and the centripetal Coriolis matrices satisfy the following relationship [22]

$\xi^{T}\left(\frac{1}{2} \dot{M}(q)-V_{m}(q, \dot{q})\right) \xi=0 \quad \forall \xi \in \mathbb{R}^{n}$

where $\dot{M}(q)$ represents the time derivative of the inertia matrix.

Property 3 The centripetal Coriolis matrix satisfies the following relationship [20]

$V_{m}(q, v) \xi=V_{m}(q, \xi) v \quad \forall \xi, v \in \mathbb{R}^{n}$.

Property 4 The norm of the centripetal Coriolis matrix, and the gravitational effects with the friction vector can be upper bounded as follows [21]

$$
\begin{aligned}
\left\|V_{m}(q, \xi)\right\|_{i \infty} & \leq \zeta_{c 1}\|\xi\|, \quad\left\|F_{d}\right\| \leq \zeta_{f}, \quad\|G(q)\| \\
& \leq \zeta_{g} \quad \forall \xi \in \mathbb{R}^{n}
\end{aligned}
$$

where $\zeta_{c 1}, \zeta_{f}, \zeta_{g} \in \mathbb{R}$ are known positive bounding constants and $\|\cdot\|_{i \infty}$ denotes the induced infinity norm of a matrix.

Property 5 The robot dynamics given in Eq. 1 can be linearly parameterized as follows [21]

$$
Y(q, \dot{q}, \ddot{q}) \phi_{r}=M(q) \ddot{q}+V_{m}(q, \dot{q}) \dot{q}+G(q)+F_{d} \dot{q}
$$

where $\phi_{r} \in \mathbb{R}^{p}$ contains the constant system parameters, and $Y(q, \dot{q}, \ddot{q}) \in \mathbb{R}^{n \times p}$ denotes the regression matrix that is a function of $q(t), \dot{q}(t)$ and $\ddot{q}(t)$. The formulation of Eq. 9 can be rewritten in terms of the desired trajectory and its time derivatives in the following manner

$$
\begin{aligned}
Y_{d}\left(q_{d}, \dot{q}_{d}, \ddot{q}_{d}\right) \phi_{r}= & M\left(q_{d}\right) \ddot{q}_{d}+V_{m}\left(q_{d}, \dot{q}_{d}\right) \dot{q}_{d} \\
& +G\left(q_{d}\right)+F_{d} \dot{q}_{d}
\end{aligned}
$$

Fig. 1 Tendon driven system dynamics: a general view

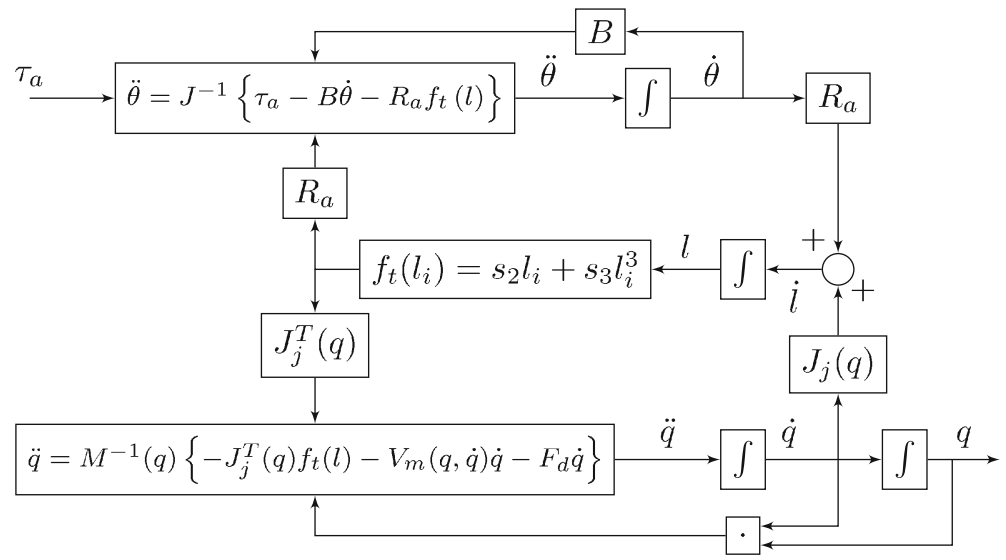


where the desired regression matrix $Y_{d}\left(q_{d}, \dot{q}_{d}, \ddot{q}_{d}\right) \in$ $\mathbb{R}^{n \times p}$ is a function of the desired link position, velocity, and acceleration vectors denoted by $q_{d}(t), \dot{q}_{d}(t)$, $\ddot{q}_{d}(t) \in \mathbb{R}^{n}$, respectively.

Property 6 The unknown actuator parameter matrices $J$ and $B$ are bounded by known upper bounds which are determined as follows

$\lambda_{\max }\{J\}<\bar{J}, \quad \lambda_{\max }\{B\}<\bar{B}$

where $\bar{J}, \bar{B} \in \mathbb{R}$ are positive constants, and $\lambda_{\max }\{\cdot\}$ denotes the maximum eigenvalue of a matrix.

Aside from the assumption that the force/ elongation characteristics of the tendons being perfectly known, our controller development and stability analysis also utilizes the following assumptions, similar to that of [5]:

Assumption $1 \operatorname{rank}\left(J_{j}(q)\right)=n$ for any $q \in \mathbb{R}^{n}$.

Assumption 2 There exists a positive valued vector of bias forces, $f_{b}$, such that $J_{j}(q)^{T} f_{b}=0$ where this bias vector is used to keep the joint tendon tension vector $f_{t}(l)$ positive without changing the joint forces generated by the tendons.

Assumption 3 Each entry of the vector $f_{t}(l)$ satisfies $f_{t i}\left(l_{i}\right)=0$ for $l_{i}<0$ and $f_{t i}\left(l_{i}\right) \geq 0, \frac{\partial f_{t i}\left(l_{i}\right)}{\partial l_{i}}>0$ for $l_{i} \geq 0$ where $i=1,2, \ldots, m$.

Assumption 4 The matrix $\frac{\partial f_{t}(l)}{\partial l} \in \mathbb{R}^{m \times m}$ is nonsingular.

\section{Controller Formulation}

The control objective is to design a link position tracking controller for the tendon-driven robot manipulator model given by Eqs. 1, 2 and 3 under the restrictive constraint that the dynamical system parameters of Eq. 1, Eq. 2 are uncertain. In the subsequent control design, we utilize full state feedback (i.e., position and velocity information). Specifically, the controller should ensure the robot links follow a desired trajectory as closely as possible, despite the uncertain robot/actuator system parameters. In order to quantify the control objective, we define the link position tracking error $e(t) \in \mathbb{R}^{n}$ as follows

$e \triangleq q_{d}-q$

where it is assumed that the desired link position, $q_{d}(t)$ and its time derivatives are sufficiently smooth and bounded functions of time. To facilitate the subsequent control development, we define the filtered tracking error $r(t) \in \mathbb{R}^{n}$ as

$r \triangleq \dot{e}+\alpha e$

where $\alpha \in \mathbb{R}^{n \times n}$ is a positive definite, diagonal control gain matrix. In addition, to provide a method of quantifying robustness, we define the difference between the actual and estimated parameters as follows

$\widetilde{\phi}_{r} \triangleq \phi_{r}-\widehat{\phi}_{r}$

where $\widetilde{\phi}_{r}(t) \in \mathbb{R}^{p}$ represents the parameter estimation error vector and $\widehat{\phi}_{r} \in \mathbb{R}^{p}$ represents the constant best guess estimates of $\phi_{r}$ defined in Eq. 9. The backstepping based controller design procedure enforces us to define two additional auxiliary error terms $\eta_{f}(t)$, $\eta_{\theta}(t) \in \mathbb{R}^{m}$ as

$\eta_{f} \triangleq f_{t}-f_{d}$

$\eta_{\theta} \triangleq \dot{\theta}-\bar{\theta}_{d}$

where $f_{t}(t), \dot{\theta}(t)$ were defined in Eqs. 1 and 2, respectively, and $f_{d}(t), \bar{\theta}_{d}(t)$ are yet to be designed auxiliary control inputs.

Taking the time derivative of Eq. 13, premultiplying the resultant equation by $M(q)$, adding/subtracting $Y_{d} \phi_{r}, J_{j}^{T}(q) f_{d}$ and $V_{m}(q, \dot{q}) r$ to the right hand side of the resulting equation, we obtain

$$
\begin{aligned}
M(q) \dot{r}= & -V_{m}(q, \dot{q}) r+\chi+d_{1}+J_{j}^{T}(q) \eta_{f} \\
& +J_{j}^{T}(q) f_{d}+Y_{d} \phi_{r}
\end{aligned}
$$

where $\chi(q, \dot{q}, t) \in \mathbb{R}^{n}$ is an auxiliary term defined as

$$
\begin{aligned}
\chi= & M(q)\left(\ddot{q}_{d}+\alpha \dot{e}\right)-M\left(q_{d}\right) \ddot{q}_{d} \\
& +V_{m}(q, \dot{q})\left(\dot{q}_{d}+\alpha e\right)-V_{m}\left(q_{d}, \dot{q}_{d}\right) \dot{q}_{d} \\
& +G(q)-G\left(q_{d}\right)+F_{d} \dot{q}-F_{d} \dot{q}_{d} .
\end{aligned}
$$

Based on Properties 1 and 4, and using Eqs. 12 and 13, it can be proven that (see Appendix for details)

$\|\chi\| \leq \rho_{1}(\|z\|)\|z\|$

where $z(t) \in \mathbb{R}^{2 n}$ is defined as

$z(t) \triangleq\left[e^{T}(t) r^{T}(t)\right]^{T}$ 
and $\rho_{1}(\cdot) \in \mathbb{R}$ is a known positive bounding function. From the structure of Eq. 17 and the subsequent stability analysis, we design $f_{d}(t)$ in the following form

$$
f_{d}=\left(J_{j}^{T}(q)\right)^{+}\left(-K_{r} r-k_{n}\left(\rho_{1}^{2}(r, e)+\rho_{2}^{2}\right) r-e-Y_{d} \hat{\phi}_{r}\right)
$$

where $(\cdot)^{+}$is used to represent the pseudo inverse of a matrix [19], $K_{r} \in \mathbb{R}^{n \times n}$ is a constant, positive definite, diagonal gain matrix, $k_{n} \in \mathbb{R}$ is a constant positive damping gain, $\hat{\phi}_{r}$ was defined in Eq. 14, and $\rho_{2} \in \mathbb{R}$ is a positive bounding constant designed to satisfy

$\rho_{2} \geq\|\widetilde{F}\|$

where $\widetilde{F}(t) \triangleq Y_{d} \phi_{r}-Y_{d} \hat{\phi}_{r}+d_{1} \in \mathbb{R}^{n}$. Inserting Eqs. 21 into 17, the closed-loop dynamics for the filtered tracking error term $r(t)$ is obtained to have the following form

$$
\begin{aligned}
M(q) \dot{r}= & -V_{m}(q, \dot{q}) r+\chi+\widetilde{F}+J_{j}^{T}(q) \eta_{f}-K_{r} r \\
& -k_{n}\left(\rho_{1}^{2}(r, e)+\rho_{2}^{2}\right) r-e .
\end{aligned}
$$

From Eq. 23, it can be seen that to ensure the stability and convergence of the tracking error signal, we require the dynamics of the auxiliary signal $\eta_{f}(t)$. To this end, we take the time derivative of Eq. 15 and make use of Eqs. 1, 3, 13 and 21, to produce

$\dot{\eta}_{f}=\Omega_{1}+\Omega_{2}+\frac{\partial f_{t}(l)}{\partial l} R_{a} \dot{\theta}$

where the right hand side is segregated into the auxiliary variables $\Omega_{1}(q, \dot{q}, l, t) \in \mathbb{R}^{m}$ containing known and measurable parameters, and $\Omega_{2}\left(q, \dot{q}, l, \phi_{r}, t\right) \in$ $\mathbb{R}^{m}$ containing uncertain system parameters, and are explicitly defined as

$$
\begin{aligned}
\Omega_{1} \triangleq & \frac{\partial f_{t}(l)}{\partial l} J_{j}(q) \dot{q}+\frac{d}{d t}\left\{\left(J_{j}^{T}(q)\right)^{+} Y_{d} \hat{\phi}_{r}\right\} \\
& -\frac{d}{d t}\left\{\left(J_{j}^{T}(q)\right)^{+}\right\}\left(-K_{r} r-k_{n}\left(\rho_{1}^{2}+\rho_{2}^{2}\right) r-e\right) \\
+ & \left(J_{j}^{T}(q)\right)^{+}\left\{\dot{e}+2 k_{n} \rho_{1} r \frac{\partial \rho_{1}}{\partial e} \dot{e}\right. \\
+ & {\left[K_{r}+k_{n}\left(\rho_{1}^{2}+\rho_{2}^{2}\right) I_{n}\right.} \\
& \left.\left.+2 k_{n} r\left(\rho_{1} \frac{\partial \rho_{1}}{\partial r}\right)\right]\left(\ddot{q}_{d}+\alpha \dot{e}\right)\right\}
\end{aligned}
$$

and

$$
\begin{gathered}
\Omega_{2} \triangleq\left(J_{j}^{T}(q)\right)^{+}\left\{\left[K_{r}+k_{n}\left(\rho_{1}^{2}+\rho_{2}^{2}\right) I_{n}+2 k_{n} r\left(\rho_{1} \frac{\partial \rho_{1}}{\partial r}\right)\right]\right. \\
\times\left[M ^ { - 1 } ( q ) \left(J_{j}^{T}(q) f_{t}(l)+V_{m}(q, \dot{q}) \dot{q}+F_{d} \dot{q}\right.\right. \\
\left.\left.\left.+G(q)+d_{1}\right)\right]\right\} .
\end{gathered}
$$

Notice that all the entries of Eq. 25 are known and/or measurable signals while Eq. 26 contains uncertain system parameters, therefore cannot be directly used in the controller design. At this stage, motivated to ensure the convergence of $\eta_{f}(t)$, we add/subtract $\frac{\partial f_{t}(l)}{\partial l} R_{a} \bar{\theta}_{d}$ term to the right hand side of Eq. 24 to obtain

$\dot{\eta}_{f}=\Omega_{1}+\Omega_{2}+\frac{\partial f_{t}(l)}{\partial l} R_{a} \eta_{\theta}+\frac{\partial f_{t}(l)}{\partial l} R_{a} \bar{\theta}_{d}$

where Eq. 16 was utilized. From the structure of Eq. 27 and the subsequent stability analysis, we design the auxiliary signal $\bar{\theta}_{d}(t)$ in the following form

$\bar{\theta}_{d}=\Lambda\left(-K_{f} \eta_{f}-J_{j}(q) r-\Omega_{1}-\widehat{\Omega}_{2}-v_{R 1}\right)$

where the auxiliary variable $\Lambda(l) \in \mathbb{R}^{m \times m}$ is defined as

$\Lambda \triangleq\left(\frac{\partial f_{t}(l)}{\partial l} R_{a}\right)^{-1}$,

$K_{f}$ is a positive definite, constant, diagonal gain matrix, $\Omega_{1}(\cdot)$ was defined in Eq. 25, $\widehat{\Omega}_{2}\left(\hat{\phi}_{r}, t\right)$ is the estimate of $\Omega_{2}\left(\phi_{r}, t\right)$ with $\hat{\phi}_{r}$ being the constant best guess estimates of $\phi_{r}$, and $v_{R 1}(t)$ is a differentiable robust term defined in the following form [23]

$v_{R 1}=\frac{\rho_{3}^{2}}{\epsilon_{1}} \eta_{f}$

where $\epsilon_{1} \in \mathbb{R}$ is a positive constant, and $\rho_{3}(e, r, l, t) \in \mathbb{R}$ is a positive bounding function designed to satisfy

$\rho_{3}(e, r, l, t) \geq\left\|\widetilde{\Omega}_{2}\right\|$

with $\widetilde{\Omega}_{2} \triangleq \Omega_{2}-\widehat{\Omega}_{2}$.

After substituting Eqs. 28 into 27, the closed-loop dynamics for the auxiliary tracking error signal $\eta_{f}(t)$ is obtained to have the following form

$\dot{\eta}_{f}=-K_{f} \eta_{f}-J_{j}(q) r+\frac{\partial f_{t}(l)}{\partial l} R_{a} \eta_{\theta}+\widetilde{\Omega}_{2}-v_{R 1}$. 
At this stage, the backstepping design procedure requires the dynamics of $\eta_{\theta}(t)$, which can be obtained by taking the time derivative of Eq. 16, inserting Eq. 28 then pre-multiplying both sides of the resulting equation by $J$ and applying Eqs. 1, 2 and 3 as

$J \dot{\eta}_{\theta}=\Omega_{3}+\Omega_{4}+\tau_{a}$

where the right hand side is segregated into the auxiliary terms $\Omega_{3}(q, \dot{q}, l, \dot{\theta}, t) \in \mathbb{R}^{m}$ containing measurable/known variables, and $\Omega_{4}\left(q, \dot{q}, l, \dot{\theta}, \phi_{r}, J, B, t\right) \in$ $\mathbb{R}^{m}$ containing uncertain system parameters, and are explicitly defined as follows

$$
\begin{aligned}
\Omega_{3} \triangleq & -R_{a} f_{t}(l)+J \frac{d \Lambda}{d t}\left(K_{f} \eta_{f}+J_{j}(q) r+\Omega_{1}\right. \\
& \left.+\widehat{\Omega}_{2}+v_{R 1}\right)+J \Lambda\left\{K_{f}\left(\Omega_{1}+\frac{\partial f_{t}(l)}{\partial l} R_{a} \dot{\theta}\right)\right. \\
& +\frac{d}{d t}\left\{J_{j}(q)\right\} r+J_{j}(q)\left(\ddot{q}_{d}+\alpha \dot{e}\right) \\
& +\frac{\partial}{\partial q}\left(\Omega_{1}+\widehat{\Omega}_{2}+v_{R 1}\right) \dot{q} \\
& +\frac{\partial}{\partial l}\left(\Omega_{1}+\widehat{\Omega}_{2}+v_{R 1}\right)\left(J_{j}(q) \dot{q}+R_{a} \dot{\theta}\right) \\
& \left.+\frac{\partial}{\partial t}\left(\Omega_{1}+\widehat{\Omega}_{2}+v_{R 1}\right)\right\}
\end{aligned}
$$

and

$$
\begin{aligned}
\Omega_{4} \triangleq & -B \dot{\theta}-d_{2}+J \Lambda\left\{K_{f} \Omega_{2}\right. \\
- & \left(J_{j}(q)-\frac{\partial}{\partial \dot{q}}\left(\Omega_{1}+\widehat{\Omega}_{2}+v_{R 1}\right)\right) \\
\times & {\left[M ^ { - 1 } ( q ) \left(J_{j}^{T}(q) f_{t}(l)+V_{m}(q, \dot{q}) \dot{q}\right.\right.} \\
& \left.\left.\left.+F_{d} \dot{q}+G(q)+d_{1}\right)\right]\right\} .
\end{aligned}
$$

Based on the previous development and the ensuing stability analysis, we now design the control torque input signal $\tau_{a}(t)$ as follows

$\tau_{a}=-K_{\theta} \eta_{\theta}-\left(\frac{\partial f_{t}(l)}{\partial l} R_{a}\right)^{T} \eta_{f}-\Omega_{3}-\widehat{\Omega}_{4}-v_{R 2}$

where, similar to the design of Eq. 28, $K_{\theta} \in \mathbb{R}^{m \times m}$ is a positive definite, constant, diagonal gain matrix, $\Omega_{3}$ was defined in Eq. $34, \widehat{\Omega}_{4}\left(\hat{\phi}_{r}, \hat{J}, \hat{B}, t\right) \in \mathbb{R}^{m}$ is the estimate of $\Omega_{4}\left(\phi_{r}, J, B, t\right)$ with ( $(\cdot)$ being used to illustrate the constant best guess estimates of (.), and $v_{R 2}(t)$ is the robust term defined in the following form [23]

$v_{R 2}=\frac{\rho_{4}^{2}}{\epsilon_{2}} \eta_{\theta}$

where $\epsilon_{2} \in \mathbb{R}$ is a positive constant, and $\rho_{4}\left(e, r, l, \eta_{\theta}, t\right) \in \mathbb{R}$ is a positive bounding function designed to satisfy

$\rho_{4}\left(e, r, l, \eta_{\theta}, t\right) \geq\left\|\widetilde{\Omega}_{4}\right\|$

with $\widetilde{\Omega}_{4} \triangleq \Omega_{4}-\widehat{\Omega}_{4} \in \mathbb{R}^{m}$. After substituting the control torque input given by Eqs. 36 into 33, we obtain the closed-loop error dynamics for $\eta_{\theta}(t)$ as shown below

$J \dot{\eta}_{\theta}=-K_{\theta} \eta_{\theta}-\left(\frac{\partial f_{t}(l)}{\partial l} R_{a}\right)^{T} \eta_{f}+\widetilde{\Omega}_{4}-v_{R 2}$.

A schematic representation of the controller formulation is presented in Fig. 2. Having formed the closed-loop dynamics for the error signals $r(t), \eta_{f}(t)$ and $\eta_{\theta}(t)$, we are now ready to analyze the stability of the overall system and the convergence of the link position tracking error $e(t)$.
Fig. 2 An illustration of the controller formulation

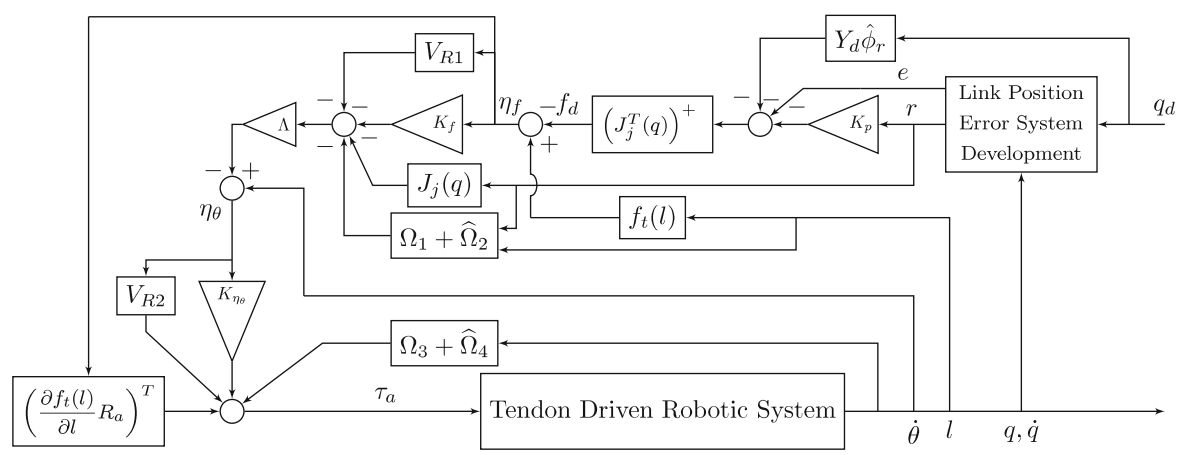




\section{Stability Analysis}

The stability of the closed-loop system will be investigated by utilizing Lyapunov-based arguments. Following theorem formalizes the stability analysis.

Theorem 1 The robust controller of Eq. 36 and the auxiliary control inputs (21) and (28) with the robust terms (30) and (37) guarantees uniform ultimate boundedness of the link position tracking error $e(t)$ in the sense that

$$
\|e(t)\| \leq \sqrt{\frac{a}{b}\|x(0)\|^{2} \exp (-\beta t)+\frac{2 \epsilon}{b \beta}(1-\exp (-\beta t))}
$$

where $x(t) \triangleq\left[\begin{array}{llll}e^{T} & r^{T} & \eta_{f}^{T} & \eta_{\theta}^{T}\end{array}\right]^{T} \in \mathbb{R}^{2(n+m) \times 1}$ is the combined error signal, $a, b, \beta, \epsilon \in \mathbb{R}$ are positive scalars defined explicitly as

$a \triangleq \max \left\{1, m_{2}, \bar{J}\right\}$

$b \triangleq \min \left\{1, m_{1}, \lambda_{\min }(J)\right\}$

$\beta \triangleq \frac{2 \min \left\{\min \left\{\lambda_{\min }\left(K_{r}\right), \lambda_{\min }(\alpha)\right\}-\frac{1}{4 k_{n}}, \lambda_{\min }\left(K_{f}\right), \lambda_{\min }\left(K_{\theta}\right)\right\}}{\max \left\{1, m_{2}, \bar{J}\right\}}$

$\epsilon \triangleq \frac{1}{4 k_{n}}+\epsilon_{1}+\epsilon_{2}$

where $m_{1}, m_{2}, \bar{J}, \epsilon_{1}, \epsilon_{2}, k_{n}, K_{f}, K_{\theta}$ and $K_{r}$ were previously defined, and the notation $\lambda_{\min }(\cdot)$ is used to denote the minimum eigenvalue of a matrix.

Proof To prove the Theorem, we start by defining a non-negative scalar function of the form

$V=\frac{1}{2} r^{T} M r+\frac{1}{2} e^{T} e+\frac{1}{2} \eta_{f}^{T} \eta_{f}+\frac{1}{2} \eta_{\theta}^{T} J \eta_{\theta}$

which can be bounded from below and above as

$\frac{1}{2} \min \left\{1, m_{1}, \lambda_{\min }(J)\right\}\|x\|^{2} \leq V \leq \frac{1}{2} \max \left\{1, m_{2}, \bar{J}\right\}\|x\|^{2}$.
Taking the time derivative of Eq. 45 along Eqs. 23, 13, 32 and 39, making use of Eq. 6, and then canceling common terms, we obtain

$$
\begin{aligned}
\dot{V}= & -r^{T} K_{r} r-e^{T} \alpha e-\eta_{f}^{T} K_{f} \eta_{f}-\eta_{\theta}^{T} K_{\theta} \eta_{\theta} \\
& +r^{T}\left[\chi-k_{n} \rho_{1}^{2} r\right]+r^{T}\left[\widetilde{F}-k_{n} \rho_{2}^{2} r\right] \\
& +\eta_{f}^{T}\left[\widetilde{\Omega}_{2}-v_{R 1}\right]+\eta_{\theta}^{T}\left[\widetilde{\Omega}_{4}-\frac{\rho_{4}^{2}}{\epsilon_{2}} \eta_{\theta}\right] .
\end{aligned}
$$

Note that using the definition given in Eq. 30 the $\eta_{f}^{T}\left[\widetilde{\Omega}_{2}-v_{R 1}\right]$ term can be bounded as follows [23]

$$
\begin{aligned}
\eta_{f}^{T}\left[\tilde{\Omega}_{2}-v_{R 1}\right] & \leq \rho_{3}\left\|\eta_{f}\right\|-\frac{\rho_{3}^{2}\left\|\eta_{f}\right\|^{2}}{\epsilon_{1}} \\
& \leq \rho_{3}\left\|\eta_{f}\right\|\left(1-\frac{\rho_{3}\left\|\eta_{f}\right\|}{\epsilon_{1}}\right) \\
& \leq \epsilon_{1} .
\end{aligned}
$$

After applying Eqs. 19, 22, 38 and 48, the right hand side of Eq. 47 can be upper bounded to have the following form

$$
\begin{aligned}
\dot{V} \leq & -\lambda_{\min }\left(K_{r}\right)\|r\|^{2}-\lambda_{\min }(\alpha)\|e\|^{2}-\lambda_{\min }\left(K_{f}\right)\left\|\eta_{f}\right\|^{2} \\
& -\lambda_{\min }\left(K_{\theta}\right)\left\|\eta_{\theta}\right\|^{2} \\
& +\left[\rho_{1}\|z\|\|r\|-k_{n} \rho_{1}^{2}\|r\|^{2}\right] \\
& +\left[\rho_{2}\|r\|-k_{n} \rho_{2}^{2}\|r\|^{2}\right] \\
& +\left[\rho_{4}\left\|\eta_{\theta}\right\|-\frac{\rho_{4}^{2}\left\|\eta_{\theta}\right\|^{2}}{\epsilon_{2}}\right]+\epsilon_{1} .
\end{aligned}
$$

Applying the nonlinear damping argument (i.e., first by adding and subtracting $1 / 4 k_{n}$ and then completing the squares) to the first and second bracketed terms of Eq. 49, the right hand side can further be bounded as

$$
\begin{aligned}
\dot{V} \leq & -\left(\min \left\{\lambda_{\min }\left(K_{r}\right), \lambda_{\min }(\alpha)\right\}-\frac{1}{4 k_{n}}\right)\|z\|^{2} \\
& -\lambda_{\min }\left(K_{f}\right)\left\|\eta_{f}\right\|^{2}-\lambda_{\min }\left(K_{\theta}\right)\left\|\eta_{\theta}\right\|^{2} \\
& +\left[\rho_{4}\left\|\eta_{\theta}\right\|-\frac{\rho_{4}^{2}\left\|\eta_{\theta}\right\|^{2}}{\epsilon_{2}}\right]+\epsilon_{1}+\frac{1}{4 k_{n}} .
\end{aligned}
$$

where $z(t)$ was previously defined in Eq. 20. Finally, using a similar manipulation to that of Eq. 48 to the bracketed term of Eq. 50, we obtain

$$
\begin{aligned}
\dot{V} \leq & -\min \left\{\min \left\{\lambda_{\min }\left(K_{r}\right), \lambda_{\min }(\alpha)\right\}\right. \\
& \left.-\frac{1}{4 k_{n}}, \lambda_{\min }\left(K_{f}\right), \lambda_{\min }\left(K_{\theta}\right)\right\}\|x\|^{2}+\epsilon
\end{aligned}
$$


where $\epsilon$ was previously defined in Eq. 44. Using the definition of $x(t)$ and the upper bound of $V(t)$ given in Eq. 46, the upper bound of $\dot{V}(t)$ given (51) can be reformulated as

$\dot{V} \leq-\beta V+\epsilon$

where $\beta$ was defined in Eq. 43. The solution of the above differential inequality yields

$V(t) \leq V(0) \exp (-\beta t)+\frac{\epsilon}{\beta}(1-\exp (-\beta t))$

and from Eq. 46, we can obtain the following upper bound for $x(t)$

$\|x(t)\| \leq \sqrt{\frac{a}{b}\|x(0)\|^{2} \exp (-\beta t)+\frac{2 \epsilon}{b \beta}(1-\exp (-\beta t))}$

where $a$ and $b$ were previously defined in Eqs. 41 and 42 , respectively. Based on the definition of $x(t)$ and Eq. 54, it can be shown that the tracking error term $e(t)$ is bounded as stated in Eq. 40. Moreover after applying standard signal chasing arguments, we can show that all signals in the closed-loop system are bounded.

Remark 1 In the controller design it was assumed that the force/elongation characteristic of the tendon is perfectly known. We would like to mention that the proposed robust controller can be modified to compensate for simplistic tendon transmission models (i.e. linear). However as presented in [4-6] the actual tendon characteristics includes highly nonlinear terms as hysteresis. We would like to point out that in an actual implementation due to the robust nature of proposed controller, some of these uncertainties might be compensated by readjusting the controller gains upto a point. Still additional uncertainties like the elastic tendon characteristics would result in higher tracking errors in an actual implementation.

\section{Simulation Studies}

To illustrate the performance of the proposed controller we have performed simulation studies on a two link robotic arm driven by 6 tendons as shown in Fig. 3.
The dynamical parameters of the robot used in our simulation studies has the following model matrices

$$
\begin{aligned}
M & =\left[\begin{array}{cc}
p_{1}+2 p_{3} \cos \left(q_{2}\right) & p_{2}+p_{3} \cos \left(q_{2}\right) \\
p_{2}+p_{3} \cos \left(q_{2}\right) & p_{2}
\end{array}\right] \\
V_{m} & =\left[\begin{array}{cc}
-p_{3} \sin \left(q_{2}\right) \dot{q}_{2} & -p_{3} \sin \left(q_{2}\right)\left(\dot{q}_{1}+\dot{q}_{2}\right) \\
p_{3} \sin \left(q_{2}\right) \dot{q}_{1} & 0
\end{array}\right] \\
F_{d} & =\left[\begin{array}{cc}
f_{d 1} & 0 \\
0 & f_{d 2}
\end{array}\right]
\end{aligned}
$$

and the Jacobian matrix is defined as follows [5],

$J_{j}=s_{1}\left[\begin{array}{cccccc}1 & -1 & 1 & -1 & 1 & -1 \\ 0 & 0 & -1 & 1 & 1 & -1\end{array}\right]^{T}$.

Tendon tensile forces can be calculated as a function of tendon expansions in the following manner [5],

$f_{t, i}\left(l_{i}\right)=\left\{\begin{array}{cc}s_{2} l_{i}+s_{3} l_{i}^{3} & l_{i} \geqslant 0 \\ 0 & l_{i}<0\end{array}\right.$

for $i=1, \ldots, 6$ where $l_{i}$ are the tendon expansions and $f_{t, i}\left(l_{i}\right)$ are the members of the vector of the tendon tensile forces which is defined as follows

$f_{t}(l)=\left[f_{t, 1}\left(l_{1}\right) f_{t, 2}\left(l_{2}\right) f_{t, 3}\left(l_{3}\right) f_{t, 4}\left(l_{4}\right) f_{t, 5}\left(l_{5}\right) f_{t, 6}\left(l_{6}\right)\right]^{T}$.

The robot parameters were taken as $p_{1}=$ $0.006 \mathrm{kgm}^{2}, p_{2}=0.003 \mathrm{kgm}^{2}, p_{3}=0.002 \mathrm{kgm}^{2}$, $f_{d 1}=0.005 \mathrm{Nm} \mathrm{sec}, f_{d 2}=0.001 \mathrm{Nm} \mathrm{sec}, s_{1}=$ $0.015, s_{2}=7907.5 \mathrm{~N} / \mathrm{m}$ and $s_{3}=1.7898 \times$ $10^{8} \mathrm{~N} / \mathrm{m}^{3}$ for the simulations. We would like to note that most of the system and tendon parameters used in our simulation studies are taken from [5]. The actuator part of the tendon mechanism has identical six dc motors which have the inertia of $10 \mathrm{gcm}^{2}$ and viscous friction of $0.25 \times 10^{-3} \mathrm{Nm} \mathrm{sec} / \mathrm{rad}$, each actuator is assumed to have a gearbox mechanism having $1 / 32$ gear ratio and the pulleys mounted on the actuators have a radius of $10 \mathrm{~cm}$. During the numerical studies the best guessed estimates of the system parameters are selected to be 80 percent of the actual system parameters.

The desired trajectory of the robot is selected as $q_{d 1}=q_{d 2}=0.5 \sin (0.5 t)\left(1-\exp \left(-0.3 t^{3}\right)\right) \mathrm{rad}$ with the initial positions of each link being set to $0.5 \mathrm{rad}$. To ease the tuning process an auxiliary gain $K_{p}=K_{r}+k_{n}\left(\rho_{1}^{2}+\rho_{2}^{2}\right)$ was taken and assumed 
Fig. 3 Two link planar robot driven by six tendons

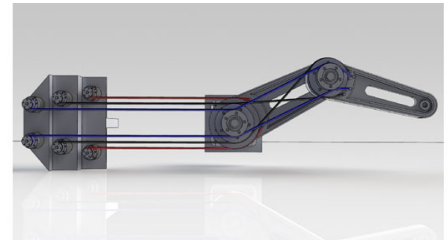

(a) Top view

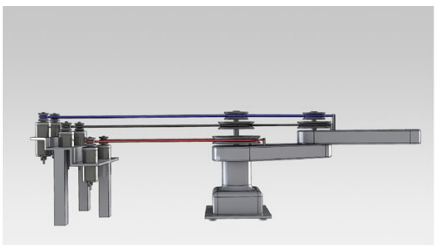

(b) Side view

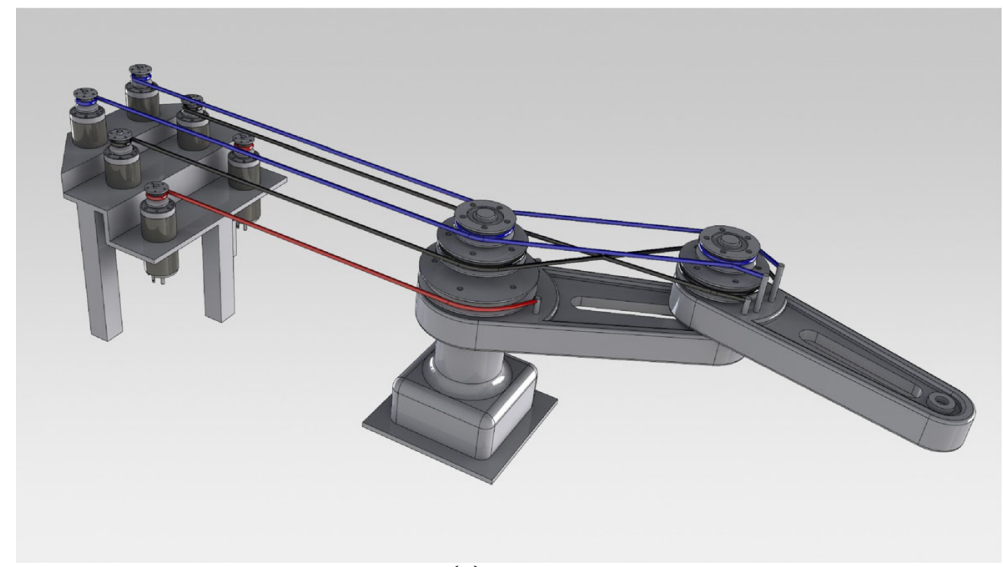

(c) Overall

to be a constant gain matrix. In view of Eq. 51, the controller gains were selected to ensure that

$\min \left(\lambda_{\min }\left(K_{r}\right), \lambda_{\min }(\alpha)\right)-\frac{1}{4 k_{n}}>0$

is satisfied. Controller gain matrices are selected as $\alpha=\operatorname{diag}\{8,10\}, K_{p}=\operatorname{diag}\{2.8,2.6\}, K_{\theta}=0.1 I_{2}$, $K_{f}=5 I_{2}, \rho_{3}=1, \rho_{4}=4$ and $\epsilon_{1}=\epsilon_{2}=0.01$. As can be seen from the selection of controller gains, superficially tuning of the controller gains is enough to prove the validity of the controller algorithm nevertheless accurate tuning of the controller gains may increase the controller performance.

The simulation results are shown in Figs. 4, 5, and 6. Figure 4 shows the link position tracking errors in degrees and in inner figure the enlarged steady state error signals can be seen. Figure 5 presents the control torques applied to each actuators, while Fig. 6 presents the each tendon tensile forces. As can be viewed from Fig. 4 after a very short period of time (less than 5 secs.) the tracking error terms for both joint converge to small values. During this time the controller inputs
Fig. 4 Position tracking errors for each link
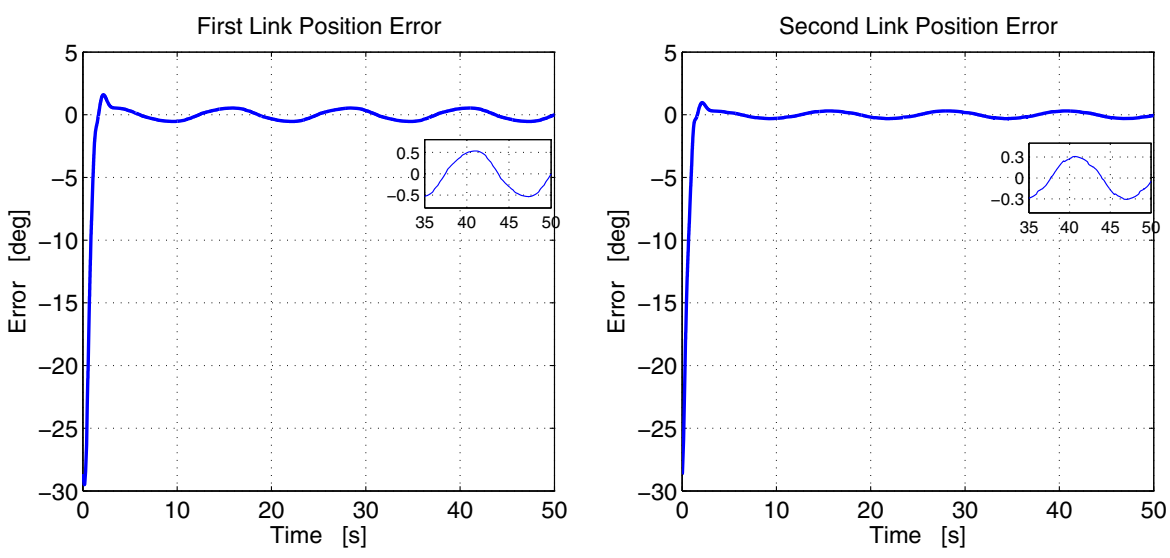
Fig. 5 Control input torques

Fig. 6 Tendon tensile forces

(see Fig. 5) with the tendon tensile forces (see Fig. 6) are in acceptable regions.

\section{Concluding Remarks}

In this work, we have presented a full state feedback, nonlinear robust controller for tendon-driven robotic mechanisms subject to parametric uncertainty
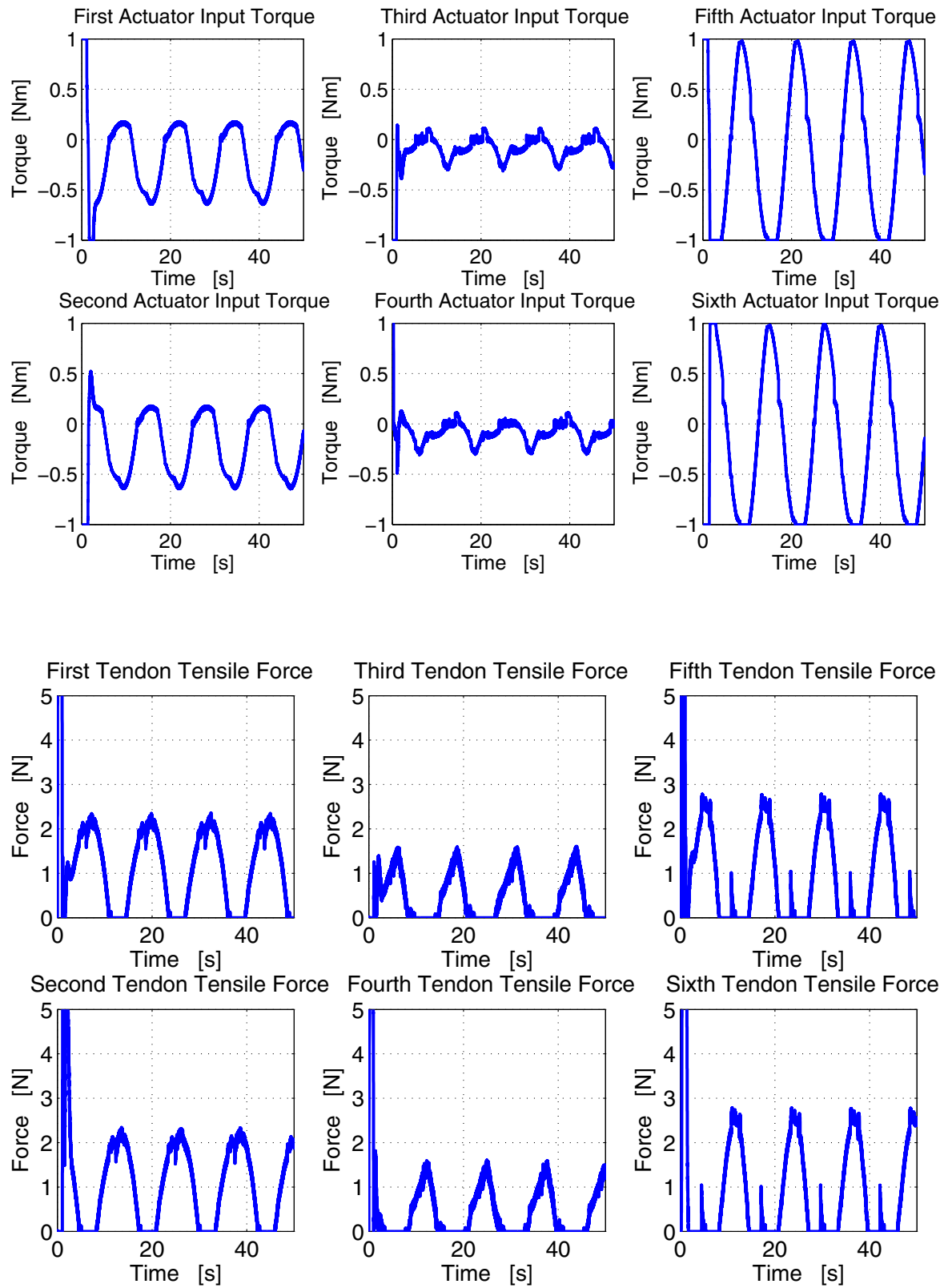

in system dynamics. Despite the lack of exact knowledge of system parameters and presence of external disturbances, the proposed controller ensured practical trajectory tracking in the sense that; the norm of the link position tracking error signal is forced to enter an ultimate bound in finite time. Stability of the closed-loop system and boundedness of system states are proven via Lyapunov based arguments. Simulation studies are performed to illustrate the 
effectiveness and viability of the proposed method. Our controller requires the full state feedback of all system states (i.e. both position and velocity information of the robot joints and the actuators) and the exact knowledge of the tendon tensile forces generated by tendon expansions. From the illustration given Fig. 2, it is obvious that the implementation of the proposed controller is far more complex compared to most conventional controllers. However thanks to the current state of micro-controllers the controller algorithm is still implementable in real time. Future work will concentrate on the design and implementation of partial state and/or output feedback controllers that would not require the perfect knowledge of the force/elongation characteristic of the tendons.

Acknowledgments The authors would like to thank the anonymous reviewers for their invaluable criticisms and suggestions during the review process. Their comments have helped us improve the manuscript in every aspect.

\section{Appendix: Proof of the Bound of $\|\chi\|$}

For the Eq. 19 we start with the previously found bound on the same term [21] (Chapter 6 Eq: 6.2-9) as

$$
\|\chi\| \leq \zeta_{1}\|e\|+\zeta_{2}\|e\|^{2}+\zeta_{3}\|r\|+\zeta_{4}\|r\|\|e\|
$$

where $\zeta_{i}, i=1,2,3,4$ are positive bounding constants that depend on the desired trajectory and physical parameters (i.e. link mass, link length, friction coefficients, etc.). After some mathematical manipulations (61) can be written to have the following form

$$
\|\chi\| \leq\left(\zeta_{1}+\zeta_{2}\|e\|\right)\|e\|+\left(\zeta_{3}+\zeta_{4}\|e\|\right)\|r\| .
$$

when bounding functions $\zeta_{5}(e)$, and $\zeta_{6}(e)$ are selected as

$$
\begin{aligned}
& \zeta_{5}(e)=\zeta_{1}+\zeta_{2}\|e\| \\
& \zeta_{6}(e)=\zeta_{3}+\zeta_{4}\|e\|
\end{aligned}
$$

we can further upper bound (62) as

$$
\|\chi\| \leq \max \left\{\zeta_{5}(e), \zeta_{6}(e)\right\}\|z\|
$$

from Eq. 64 it is clear that the bound given in Eq. 19 is satisfied via a proper selection of the bounding function $\rho_{1}(\|z\|)$ differentiable at $e=0$.

\section{References}

1. Jacobsen, S.C., Ko, H., Iverson, E.K., Davis, C.C.: Antagonistic control of a tendon driven manipulator. In: Proceedings of the IEEE International Conference on Robotics and Automation, pp. 1334-1339 (1989)

2. Kaneko, M., Paetsch, W., Tolle, H.: Input-dependent stability of joint torque control of tendon-driven robot hands. IEEE Tr. Ind. Electron 39(2), 96-104 (1992)

3. Mason, M.T., Salisbury Jr., J.K.: Robot Hands and the Mechanics of Manipulation. Cambridge, MIT Press (1985)

4. Ma, S., Hirose, S., Yashinada, H.: Design and experiments for a coupled tendon-driven manipulator. IEEE Control Syst. Mag. 13(1), 30-36 (1993)

5. Kobayashi, H., Ozawa, R.: Adaptive neural network control of tendon driven mechanisms with elastic tendons. Automatica 39, 1509-1519 (2003)

6. Chang, S., Lee, J., Yen, H.: Kinematic and compliance analysis for tendon driven robotic mechanisms with flexible tendons. Mech. Mach. Theory 40, 728-739 (2005)

7. Jacobsen, S.C., Wood, J.E., Knutti, D.F., Biggers, K.B.: The UTAH/M.I.T. dextrous hand: Work in progress. Int. J. Robot Res. 3(4), 21-50 (1984)

8. Kawamura, S., Kino, H., Won, C.: High-speed manipulation by using parallel wire-driven robots. Robotica 18(1), 18-21 (2000)

9. Lee, J.J.: Tendon-Driven Manipulators: Analysis, Synthesis and Control. Ph.D. Dissertation, Ph.D. 91-2, University of Maryland, College Park, MD, USA (1991)

10. Palli, G.: Model and control of tendon actuated robots. $\mathrm{Ph} . \mathrm{D}$. dissertation, DEIS, University of Bologna, Bologna, Italy (2007)

11. Rainey, L.W. .I, Leahy Ozawa, M.B. Jr.: Direct adaptive control for tendon-driven manipulators. In: Proceedings of the 1991 IEEE International Symposium on Intelligent Control, pp. 31-36 (1991)

12. Kobayashi, H., Hyodo, K., Ogane, D.: On tendon-driven robotic mechanisms with redundant tendons. Int. J. Robot. Res. 17(5), 561-571 (1998)

13. Potkonjak, V., Svetozarevic, B., Jovanovic, K., Holland, O.: The puller-follower control of compliant and noncompliant antagonistic tendon drives in robotic systems. Int. J. Adv. Robot Syst. 8(5), 143-155 (2011)

14. Nakayama, T., Fujimoto, H.: Trajectory tracking control of tendon driven robots using delayed force feedback. In: Proceedings of International Conference on Mechatronics. Kumamoto (2007)

15. Haiya, K., Komada, S., Hirai, J.: Control of tendondriven robotic mechanism by nonlinear springs and hysteresis characteristics. In: Proceedings of the IEEE International Conference on Mechatronics, pp. 1-6. Malaga (2009)

16. Haiya, K., Komada, S., Hirai, J.: Tension control of tendon mechanisms by compensation of nonlinear spring characteristic equation error. In: IEEE Workshop on Advanced Motion Control, pp. 42-47. Nagaoka (2010) 
17. Wimbock, T., Ott, C., Hirzinger, G.: Immersion and invariance control for an antagonistic joint with nonlinear mechanical stiffness. In: IEEE Conference on Decision and Control, pp. 1128-1135. Atlanta (2010)

18. Ozbay, U., Sahin, H.T., Zergeroglu, E.: Robust tracking control of kinematically redundant robot manipulators subject to multiple-self motion criteria. Robotica 26, 711728 (2008)

19. Rao, C.R., Mitra, S.K.: Generalized Inverse of Matrices and Its Applications. Wiley, New York (1971)
20. Nicosia, S., Tomei, P.: Robot control by using only position measurements. IEEE Tr. Autom Control 35(9), 1058-1061 (1990)

21. Lewis, F.L., Abdallah, C.T., Dawson, D.M.: Control of Robot Manipulators. Macmillan Publishing Co., New York (1993)

22. Tomei, P.: Adaptive PD controller for robot manipulators. IEEE Tr. Robot. Autom 7(4), 565-570 (1991)

23. Dawson, D.M., Bridges, M.M., Qu, Z.: Nonlinear Control of Robotic Manipulators for Environmental Waste and Restoration. Prentice Hall, Englewood Cliffs (1995) 